\title{
Creativity in problem solving: integrating two different views of insight
}

\author{
Per Øystein Haavold ${ }^{1}\left[\right.$ D . Bharath Sriraman ${ }^{2}$
}

Accepted: 24 August 2021 / Published online: 2 September 2021

(c) The Author(s) 2021

\begin{abstract}
Even after many decades of productive research, problem solving instruction is still considered ineffective. In this study we address some limitations of extant problem solving models related to the phenomenon of insight during problem solving. Currently, there are two main views on the source of insight during problem solving. Proponents of the first view argue that insight is the consequence of analytic thinking and a sequence of conscious and stepwise steps. The second view suggests that insight is the result of unconscious processes that come about only after an impasse has occurred. Extant models of problem solving within mathematics education tend to highlight the first view of insight, while Gestalt inspired creativity research tends to emphasize the second view of insight. In this study, we explore how the two views of insight-and the corresponding set of models-can describe and explain different aspects of the problem solving process. Our aim is to integrate the two different views on insight, and demonstrate how they complement each other, each highlighting different, but important, aspects of the problem solving process. We pursue this aim by studying how expert and novice mathematics students worked on two ill-defined mathematical problems. We apply both a problem solving model and a creativity model in analyzing students' work on the two problems, in order to compare and contrast aspects of insight during the students' work. The results of this study indicate that sudden and unconscious insight seems to be crucial to the problem solving process, and the occurrence of such insight cannot be fully explained by problem solving models and analytic views of insight. We therefore propose that extant problem solving models should adopt aspects of the Gestalt inspired views of insight.
\end{abstract}

Keywords Experts $\cdot$ Novices $\cdot$ Insight $\cdot$ Creativity $\cdot$ Problem solving

\section{Introduction}

Most mathematics educators would probably agree that the development of students' problem solving abilities is an important objective of instruction. Thus there has been a considerable amount of research on problem solving in the last several decades (Lester, 2013). In general, researchers into problem solving have usually defined the term problem as tasks or questions that an individual or group of individuals do not immediately know how to answer (Lester, 2013). However, this definition says very little about how to teach individuals to become better problem solvers (Lester, 2013). Several models of problem solving have therefore

\footnotetext{
Per Øystein Haavold

per.oystein.haavold@uit.no

1 UiT The Arctic University of Norway, Troms $\varnothing$, Norway

2 University of Montana, Missoula, USA
}

been developed to describe and explain factors and processes involved in problem solving - most of which have drawn heavily on Pólya's (1949) famous four-stage model of problem solving. Nevertheless, problem solving instruction is still considered ineffective. There are many reasons for this perception, but one key issue is the lack of concern for the complexity and the many factors involved in problem solving processes (Lester, 2013).

The focus of this paper is one of the more subtle yet essential factors involved in problem solving. Ever since the Gestaltists first began studying problem solving nearly 100 years ago, insight in problem solving has been of interest to psychologists (Hadamard, 1945; Ohlsson, 2011; Poincaré, 1948; Weisberg, 2015). Here, it is important to note that insight (Einsicht) within the Gestalt approach, and much of the literature on insight and problem solving, have a broader meaning than the standard definition in English. According to the Gestaltists, an individual's comprehension of a problem cannot be reduced to a collection of individual 
perceptual features. Instead, the individual perceives a particular Gestalt of the problem, which can be interpreted as the totality of the relations between its parts. Insight, to the Gestaltists, was therefore considered a mental restructuring of the problem into a more productive Gestalt (Ohlsson, 2011; Wertheimer, 1959). In this study, we draw on the Gestalt view and consider insight as a perceptual and conceptual restructuring of a problem in a more productive manner. This view of insight has also been described as mentally crossing a 'logical gap', and it has often been referred to as a sudden and unexpected feeling of comprehension during an attempt at solving a problem (Ohlsson, 2011; Sternberg \& Davidson, 1995).

Currently there are two main views on the source of insight during problem solving. Proponents of the first view argue that insight is the consequence of analytic thinking in which the problem is matched with information in memory. The solution typically unfolds in a sequence of conscious steps, and the individual has a feeling of steady incremental progress. Insight is gained gradually and consciously. The Gestaltists called this reproductive thinking (Weisberg, 2015). The second view, termed productive thinking by the Gestaltists, suggests that insight is the result of a particular set of processes distinct from conscious analytical thinking. Here, insight is the result of unconscious processes that come about only after an impasse has occurred. Furthermore, insight is gained quickly, often spontaneously, and as a result of mental restructuring of the problem (Weisberg, 2015). Extant models of problem solving within mathematics education tend to highlight the first view of insight. Lester and Kehle (2003), for example, characterizes successful problem solving as "coordinating previous experiences, knowledge, familiar representations and patterns of inference, and intuition..." (p. 510). Although unconscious processes such as intuition are sometimes mentioned, they are usually not explained or elaborated in problem solving models, and the emphasis is on analytic and conscious cognitive processes. On the other hand, within the field of creativity research and theoretical models of creativity-in particular Gestaltist inspired research-analytic thinking is considered unable to produce novelty. Highly inspired by the Gestaltists, the focus has therefore often been on more spontaneous processes that can result in a new interpretation of the problem (Weisberg, 2015).

In this study, we investigated how the two views of insight $-a$ and corresponding set of models—can describe and explain different aspects of the problem solving process. The aim of our study was to integrate the two different views on insight, and demonstrate how they complement each other, each highlighting different, but important aspects of the problem solving process. We pursued this aim by studying how expert and novice mathematics students at a large research university in Norway approached and worked on two ambiguous and ill-defined mathematical problems. We then applied both a problem solving model and a creativity model in our analysis of students' work on the two problems, in order to compare and contrast aspects of insight during the students' work. More specifically, we set out to answer the following research question:

How do expert and novice students approach and attempt to gain insight into ill-defined mathematical problems?

To work towards our aim, we made use of a novice-expert comparison, which has proven to be useful within cognitive research (National Research Council, 2000). Expertise has commonly been described as 10 years of intense preparation in some field (Ericsson \& Lehmann, 1996), or "proficiency taken to its highest level" (Glaser, 1987). However, expertise has also been defined in terms of cognitive development and knowledge structures (Hoffman, 1998), and described as a continuum or multiple stages rather than a dichotomy between experts and novices (e.g. Dreyfus \& Dreyfus, 2005). In this study, we therefore differentiated between expert and novice students according to educational background and mathematical attainment. The main rationale for this choice was to contrast mathematical performance with two different theoretical perspectives of insight during problem solving.

We also made use of ill-defined problems, which are those problems for which there are conflicting assumptions, evidence, and opinions that may lead to different solution (e.g., Kitchener, 1983; Krutetskii, 1976). They force the problem solver to deal with uncertainty, and facilitate multiple possible approaches by looking at the problem in new and productive ways. Ill-defined problems are therefore particularly useful for facilitating perceptual restructuring and insight during the problem solving process (Webb et al., 2016).

\subsection{Problem solving models}

Problem solving has long been of interest to mathematics education researchers. At the root of this research, and most problem-solving frameworks, lies the work of the eminent mathematician George Polya (Schoenfeld, 1985a). In his work How to Solve It, Pólya (1949) presented a four-step model of problem solving which consisted of the four steps understanding, planning, implementing, and looking back. The model outlines problem solving as a systematic and gradual process that facilitates insight primarily by building on prior knowledge and conscious evaluation. Because of the structured and pedagogical approach to problem solving and the explicit focus on prior knowledge, Polya's four step model has become the most popular approach to teaching and learning problem solving (Liljedahl et al., 2016).

One of the shortcomings of Polya's model is that research generated under its umbrella focused almost entirely on heuristics, or rules of thumb for making progress on difficult problems, while ignoring "managerial skills necessary to 
regulate one's activity (metacognitive skills)" (Lester, 1985, p. 62). Lester (1985) and Schoenfeld (1985a) suggested that metacognitive activity (knowledge of one's thought processes or self-regulation) underlies the application of heuristics and algorithms. As a result, Polya's model was modified (Lester, 1985; Schoenfeld, 1985a) to include a cognitive component and a metacognitive component. In the cognitive component, the four phases of understanding, planning, implementing, and looking back are labeled as orientation, organization, execution, and verification respectively. The metacognitive component consists of three classes of variables attributed to Flavell and Wellman (1977). This model purports to describe the four cognitive categories in terms of 'points' where metacognitive actions occur during problemsolving (see Fig. 1).

The cognitive component Orientation refers to strategic behavior to assess and understand a problem. It includes comprehension strategies, analysis of information, initial and subsequent representation, and assessment of familiarity and chance of success. Organization refers to identification of goals, global planning, and local planning. The category of execution refers to regulation of behavior to conform to plans. It includes performance of local actions, monitoring progress and consistency of local plans, and trade-off decisions (speed vs. accuracy). Finally, verification consists of evaluating decisions made and evaluating the outcomes of the executed plans. It includes evaluation of actions carried out in the orientation, organization, and execution categories. The metacognitive component of the model is comprised of three classes of variables, namely person variables, task variables, and strategy variables. Person variables refer to an individual's belief system and affective characteristics that may influence performance. Task variables refer to features of a task, such as the content, context, structure, syntax and process. An individual's awareness of features of a task influences performance. Finally, strategy variables are those that refer to an individual's awareness of strategies that help in comprehension, organizing, executing plans, and checking and evaluation.

The main purpose of this model is to show that metacognitive actions can influence cognitive behavior at all phases of problem solving (Lester, 1985; Schoenfeld, 1985a). The introduction of metacognitive actions is an important modification of Polya's model (Liljedahl et al., 2016). In contrast to Polya's non-specific heuristics, the introduction of metacognitive components is an acknowledgment that problem solving is an emergent process that depends on the individual's prior knowledge and internal dialogue. Unlike Pólya (1949), who prescribed heuristics applicable to all problems and problem solvers, Schoenfeld (1985a) and Lester (1985) portray problem solving heuristics as personal objects that are limited to the individual's existing knowledge and understanding of the problem (Liljedahl et al., 2016).

Nevertheless, both the original model by Pólya (1949) and the revised model (Lester, 1985; Schoenfeld, 1985a) lay out problem solving as a conscious and incremental process in which the problem solver gains insight primarily through past experience and conscious evaluation. Generally, the first step in the process, after gaining an initial understanding of the problem, would entail attempts at matching the problem with prior knowledge and evaluating whether a solution method could be transferred to the new problem. If

\section{COGNITIVE COMPONENT}

\section{METACOGNITIVE COMPONENT}

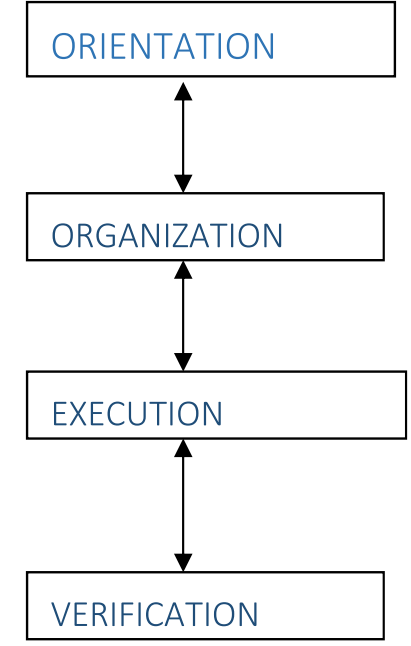

Fig. 1 The cognitive-metacognitive model (Lester, 1985)

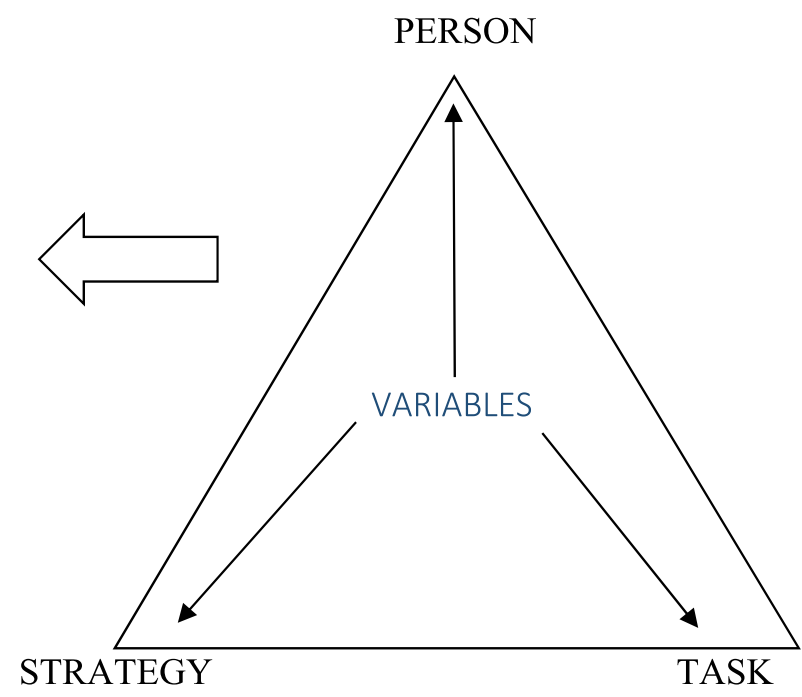


this attempt is unsuccessful, the problem solver would then move on to applying heuristic methods. Through the use of heuristics, the problem solver attempts to modify the present state of the problem so that he/she can advance towards the final goal (Weisberg, 2015). Of course, the process is not nearly this simple or linear, but it provides a general overview of the analytic approach to problem solving. Insight, or restructuring of the problem in a new and more productive manner, is gradually gained through a stepwise and conscious process.

However, within most of creativity research, which leans heavily on the Gestalt view of insight, this view of gradually gaining insight is rejected (Weisberg, 2015). Problem solving models, and similar reproductive approaches to insight in problem solving, do not explain how existing knowledge and analytic thinking can produce novel ideas, which are usually necessary for solving problems that require some form of insight. The argument is essentially that a logical system can only produce information that is already present, at least implicitly, in the premises, and that is therefore not novel (Weisberg, 2015). Therefore, insight has to be the result of some kind of special cognitive process different from the conscious and evaluative approach that characterizes analytic thinking (Ohlsson, 2011).

\subsection{Creativity models}

From the perspective of creativity research then, when one tries to solve a problem the individual will first try solutions based on similarities with other problems and consciously evaluate the progress. However, those attempts will often fail as problems that require some form of novelty will not be solved by transferring methods from similar problems. The problem solver will eventually reach an impasse. It is at this point that the person may suddenly and unconsciously gain insight through a mental restructuring of the problem and come up with a solution. This notion of insight as a result of sudden and unconscious illumination is usually attributed to the Gestalt psychologists, and it is currently the dominant view of creative thinking (Ohlsson, 2011).

According to the Gestaltists, creative thinking and insight follow a sequence of four stages, namely, preparation-incubation-illumination, and verification (Wallas, 1926; Hadamard, 1945; Poincaré, 1948). The first stage consists of working hard to understand the problem at hand. Poincaré calls this the preliminary period of conscious work. The second stage occurs when the problem is put aside for a period of time and the mind is occupied with other things. The third stage is where the solution suddenly appears while the individual is perhaps engaged in other unrelated activities. "This appearance of sudden illumination is a manifest sign of long, unconscious prior work." (Poincaré, 1948, p. 16). However, the creative process does not end here. There is a fourth and final stage, namely verification, which includes expressing the results by language or writing. At this stage one verifies the result, makes it precise, and looks for possible extensions through utilization of the result.

More recently, Ohlsson (2011) reformulated the four step Gestalt model of creativity as the insight sequence in an effort to draw a clear distinction between problem solving through analytic thinking and problem solving through sudden insight. Furthermore, while the Gestaltists were concerned with insight and creative thought on timescales of months and years, proponents of more recent Gestalt inspired research that uses the insight sequence, consider aspects of insight and creativity also on much shorter timescales (Beghetto \& Karwowski, 2019; Ohlsson, 2011). The insight sequence describes successful problem solving as a chain consisting of the following events: attempted solution $\rightarrow$ consistent failure $\rightarrow$ impasse $\rightarrow$ restructuring $\rightarrow$ insight $\rightarrow$ Solution. Unlike problem solving models that describe insight as something gained gradually through analytic and conscious thinking, the insight sequence emphasizes impasse and sudden (and unconscious) cognitive restructuring as the basis for insight (Weisberg, 2015). Presently, this restructuring is thought to occur by an impasse that causes an altered balance in a lower layer of cognitive processing systems, which leads to a new, and possibly more productive, representation in a higher and more conscious layer (Ohlsson, 2011).

An important idea in the setting of perceptual restructuring is cognitive flexibility. Cognitive flexibility refers to our ability to switch between different mental sets, tasks and strategies in light of uncertainty and impasse (Ionescu, 2012). According to Nijstad et al. (2010), cognitive flexibility is a key element for achieving creative insights, problem solutions, or ideas through the use of flexible switching among categories, approaches, and sets, and through the use of remote (rather than close) associations. Cognitive fixation, on the other hand, is the counterpart to flexibility. The notion of people struggling to come up with creative solutions because they fixate, or fail to abandon non-productive strategies, has its roots a long way back in psychological literature and features particularly in the writings of the Gestalt school (Haylock, 1987).

Although cognitive flexibility seems to relate to the intuitive concept, we still lack a clear definition and comprehension of the phenomenon (Ionescu, 2012). For example, in a review of the literature, Ionescu (2012) identified several behaviors that are considered flexible, as follows: switching between tasks or multitasking; changing behavior in light of a new rule; finding a new solution to a problem; and creating new knowledge or tools. In this paper, we consider flexibility as the ability to break away from inappropriate approaches, i.e., particular methods and strategies, within a single problem (Haylock, 1987). Regarding cognitive fixation, Haylock 
(1987) concluded that there are two particularly important types of fixation in mathematical problem solving: algorithmic fixation and content universe fixation. Algorithmic fixation is closely related to the Einstellung effect, and it refers to individuals continuing to use an initially successful algorithm or method learnt beforehand or developed through the sequence of tasks themselves. The other type of fixation, content universe fixation, refers to situations where students' thinking about mathematical problems is restricted unnecessarily to an insufficient range of elements that may be used or related to the problem (Haylock, 1987). The overcoming of these kinds of fixations, and thus allowing the mind to range over a wider set of possibilities than might first come to one's conscious awareness, is an important aspect of successful problem solving.

\subsection{Expert and novice problem solvers}

Besides the use of metacognition to describe phases of problem-solving performance, another widespread approach within the problem solving research paradigm has been to describe in detail solutions used by 'expert' problem solvers and compare this to solutions of 'novices' (Simon \& Simon, 1978). The rationale behind this genre of research was to identify strategies used by experts, and develop prescriptive models to teach students how to problem solve like experts. The main findings of studies in the 'expert-novice' genre were that experts and novices differed in their problem solving strategies because of the following:

1. Knowledge for understanding and representing problems (Orientation).

2. Strategic knowledge (Organization).

3. Repertoires of known procedures and familiar patterns (Execution and Verification).

Experts are adept at creating a representation of the problem, and understanding it in terms of fundamental principles. While experts tend to focus on structural properties of problems, novices place a greater emphasis on surface properties. Furthermore, novices are often not able to construct problem representations that are helpful in achieving solutions. This description fits into the orientation category of Lester's (1985) cognitive-metacognitive model. Experts also solve problems by using a process of successful refinements. Global planning and qualitative analysis characterize their strategies, before generating specific equations to solve the problems. Novices, on the other hand, tend to go directly from the problem text in search of equations that could be used. This behavior fits into the organization category of Lester's model. Finally, experts have developed a repertoire of problem types and solution methods besides having an extensive knowledge of basic principles. Novices are lacking much of this knowledge and experience. This observation fits into the execution and verification categories of Lester's model.

Expert and novice differences have also been studied within creativity research. In general, it is believed that the more knowledge we have in a domain, the more flexible problem solvers we are in that domain (Ionescu, 2012). The most common explanation for this aspect is that experts have acquired, over many years of practice, a vast knowledge base of techniques, methods, strategies, etc., when solving problems. This large knowledge base enables the expert often to solve novel problems by small modifications to what they already know, which in turn requires relatively minor cognitive effort (Ohlsson, 2011). However, it has also been argued that expertise could lead to less flexibility and more cognitive fixations. Expertise is generally considered to be domain specific, as skills tend to go from higher levels of generality to greater specificity as a result of practice (Ohlsson, 2011). As a result, it is conceivable that expertise can lead to less flexibility and a greater fixation on a narrow pattern of previous experiences. Others have found non-linear relationships between expertise and flexibility. In a series of clever studies on the relationship between expertise and flexibility among chess experts, Bilalic et al. (2008) found a clear difference between ordinary (3 SDs above average performance) and super experts (5 SDs above average performance). While ordinary chess experts demonstrated cognitive fixation, possibly caused by knowledge specificity, the super experts demonstrated cognitive flexibility and not fixations induced by previous mental sets. Somewhat similarly, Elgrably and Leikin (2021) recently investigated the relationship between different types of mathematical expertise and creativity. Two groups of students-expert problem solvers in mathematics and mathematics majors in university-were given a problem-posing-through investigation-task. The results showed that the expert problem solvers posed three times as many problems, with more flexible and original properties, than the mathematics majors. These findings are in line with much of the literature that indicates a clear, yet somewhat nuanced relationship between mathematical knowledge and flexibility (e.g., Haavold et al., 2020).

\section{Methods}

\subsection{Data collection and materials}

To answer our research question and work towards the aim of the study, we investigated how expert and novice mathematics students approached and attempted to gain insight into two ill-defined mathematical problems. We report here on data from task-based interviews with small (3-4) groups of students. Each session lasted for about $60 \mathrm{~min}$, in 
which the students worked on two ill-defined mathematical problems. During the interview, the interviewer answered clarification questions, but deflected more task specific and content related questions back to the students. We opted to make use of group based protocols as they are particularly appropriate for observing decision-making and students' real social cognitive behavior (Schoenfeld, 1985b).

The participants in the study consisted of two different groups of students aged 22-24 years, both of which are in their fifth and final year of their study programmes. All participants volunteered and were recruited by the first author of this paper via postings on the university's learning management system (Canvas). The first group (novice group) consisted of 12 students, divided into four groups of three, enrolled in a 5 year pre-service teacher education programme specifically aimed at teaching in primary school and lower secondary school. The students in the novice group were not mathematics specialists, and had studied only 1 year of mathematics after upper secondary school. The mathematical content in their previous mathematics studies was focused on elementary mathematical topics such as geometry, algebra, and numeracy — with a particular didactical emphasis. The expert group consisted of four master's students who excelled at graduate level mathematics. We classified this group as experts as they all were, at the time, working on their master's degree in mathematics and had demonstrated proficiency (i.e., high grades -85 th percentile) in advanced mathematics courses in calculus, number theory, algebra, and statistics.

Two ill-defined problems were given to the students. Each of them provided different types of misdirection and extensions of the problem space for the problem solvers.

Problem 1: the Roman inheritance problem The first problem comes from The Moscow Puzzles and is usually referred to as the Roman problem:

A dying Roman knowing his wife was pregnant, left a will saying that if she had a son, he would inherit two-thirds of the estate and the widow one-third, but if she had a daughter, the daughter would get one-third and the widow two-thirds. Soon after his death, his widow had twins- a boy and a girl, a possibility the will had not foreseen. What division of the estate keeps as closely as possible to the terms of the will?

There isn't a single right answer to this problem as the constraints are not fully exhaustive. This presents the students with a problem that can be repeatedly restructured and facilitate many approaches, and insight is predicated on recognizing this ambiguity. The Roman jurist, Salvian Julian, proposed for instance that the father's intent is that the daughter should receive half as much as her mother, and the son twice as much. The inheritance should be divided into seven parts, and the mother should get two parts, the son four parts, and the daughter one part. However, an opposing view is that the father wished the mother to inherit at least $1 / 3$ of the estate, but Salvian Julian would give her only 2/7. Therefore, give instead the mother $1 / 3$ and divide the rest between son and daughter according to the intended ratio of four to one. The solution of the problem depends on which of the constraints the line of reasoning is based on.

Problem 2: wrong arithmetic, but correct result The second problem was based on the idea of mathematical pathologies, which refer to examples that are specifically designed to violate properties that are perceived as valid (Sriraman \& Dickman, 2017):

Sometimes the wrong method gives us the right answer. When does this method work?

$\frac{1}{4} \times \frac{8}{5}=\frac{18}{45}$

This example is 'cooked up' knowingly to violate common properties of fraction multiplication. To gain insight into this problem, the students need to accept the counterintuitive properties as a premise and break away from established mental sets related to arithmetic. So when does this method work? One possible approach is to use algebra to identify the constraints of each digit:

$\frac{a}{b} \times \frac{c}{d}=\frac{10 a+c}{10 b+d}$

which boils down to

$a c(10 b+d)=b d(10 a+c)$

and finally

$10 a b(c-d)=c d(b-a)$

As ten is on the left side, there are now four cases that can be investigated further: $b-a=5, b-a=-5, c=5$, andd $=5$. For each of these cases, new constraints can be imposed and the situation further investigated.

\subsection{Data analysis}

Ill-defined problems contain conflicting or incomplete constraints, and they necessitate restructuring of the problem in a new and more productive manner-which is how we define insight in this paper. To identify how the students attempted to gain insight into the two ill-defined problems, we carried out a three-step analysis (e.g., Simon, 2019) in which the interviews and students' written work were analyzed retrospectively using approaches from qualitative content analysis (Mayring, 2015).

In the first step, we investigated the students' work on each problem through an inductive analysis. The goal was 
to isolate and identify each individual solution that the students attempted. We refer to this step as approaches as it includes students' solution attempts at solving the particular task, the type of strategies and reasoning employed by the students, and explicit assumptions made by the students. As we mentioned earlier, insight is predicated on some form of mental restructuring that allows the problem solver to view the problem a new and more productive manner. Although we cannot observe the cognitive processes directly, we can observe and identify the individuals' approaches, in the form of actions and utterances, which indicate how they conceive the problem's starting and goal state, constraints and operators. In other words, each approach indicates a particular mental structuring or restructuring of the problem (Weisberg, 2015).

In the second step, we made use of a mixed content analysis (Mayring, 2015) and looked more closely at the students' approaches from both creativity and problem solving perspectives. More specifically, from a problem solving perspective, we first imposed the four stages of orientation, organization, execution, and verification (Lester, 1985) on to the previously identified approaches, and examined how the students moved between approaches. This step was accomplished by further categorizing all the observed behavior, i.e., utterances and actions, for each of the identified approaches. All behavior related to assessing or understanding the problem was coded as orientation. We then coded all behavior related to organizing and execution as a common category, as it can be very difficult to distinguish planning and execution of plans (Schoenfeld, 1985a). The last category, verification, referred to all behavior related to evaluation of decisions made and the outcome of the executed plans. After the deductive coding, we made use of inductive coding with two goals in mind, as follows: (1) identify common characteristics of each phase across both problems for both groups of students respectively, and (2) identify how the groups of students moved between problem solving phases during the problem solving process.

To investigate the students' work from a creativity perspective, we made use of a creativity model based on the Gestalt view of insight in the second step of our analysis. As mentioned earlier, the Gestaltists viewed insight as dependent on sudden and cognitive restructuring (Weisberg, 2015). Although cognitive flexibility can refer to various categories and sets, in this study we considered the identified approaches as a particular mental structuring, or restructuring, of the problem. Cognitive flexibility then, in this context, becomes the ability to switch between different approaches to the ill-defined problems. Furthermore, and as Nijstad et al. (2010) point out, the use of remote associations is a particular characteristic of cognitive flexibility. Thus, we looked more closely at (1) how many different approaches the students' in each group made use of, (2) to what extent and in what way each approach differed from previous approaches in terms of strategies used and assumptions made, and 3) to what extent and in what way impasses during the problem solving process occurred-indicating the occurrence of fixations. Here, it is important to point out that we did not consider the success of each approach. It is often necessary to produce several attempts at solving an ill-defined problem in the absence of a priori knowledge of a valid solution, before finally solving it. Failed attempts are therefore often crucial to the creative process, as creative products are generated in the course of a dynamic process of exploration and assessment across both failed and successful attempts (Corazza, 2016).

In the third and final step, we attempted to develop explanatory inferences and work towards the aim of the paper. Here we compare and contrast how the two models - and corresponding views of insight—can describe and explain different aspects of the problem solving process. More specifically, we attempted to identify how and to what extent each of the two different models can describe and explain how the two groups of students gained, or failed to gain, insight into the ill-defined problems.

\section{Results}

\subsection{Problem 1: the Roman inheritance problem}

Expert students The expert group approached the problem in two ways. At the start of the first approach, the students read the problem several times, first individually and then aloud, and discussed what they were "supposed to actually find out" as one student said. Simultaneously, they wrote down some of the constraints that they had identified in the problem: the wife should get more than the daughter, but less than the son. They then quickly reasoned what the wife's proportion of the will would be if the total sum were halved. As one student said, "the wife should get exactly half of one third plus two third". They concluded the wife should get half, and the rest be split between the daughter and the son. However, they quickly concluded that this was incorrect as this would either leave the son with less than the wife, or an inheritance exceeding the upper limit.

After rejecting the first approach, the expert students made a second attempt at solving the problem. They went back to talking about the information and conditions of the problem. They then decided to set up an equation, as this would "impose the all the necessary conditions on to the problem and we can solve it" as one student said. The right side of the equation had to be 1 , as this represented the entire inheritance. The mother's share was set as $\mathrm{x}$, the son as $\mathrm{y}$ and the daughter $\mathrm{z}$. They then substituted the variables and solved the equation (see Fig. 2). 
Fig. 2 Experts' equation solution to the Roman problem

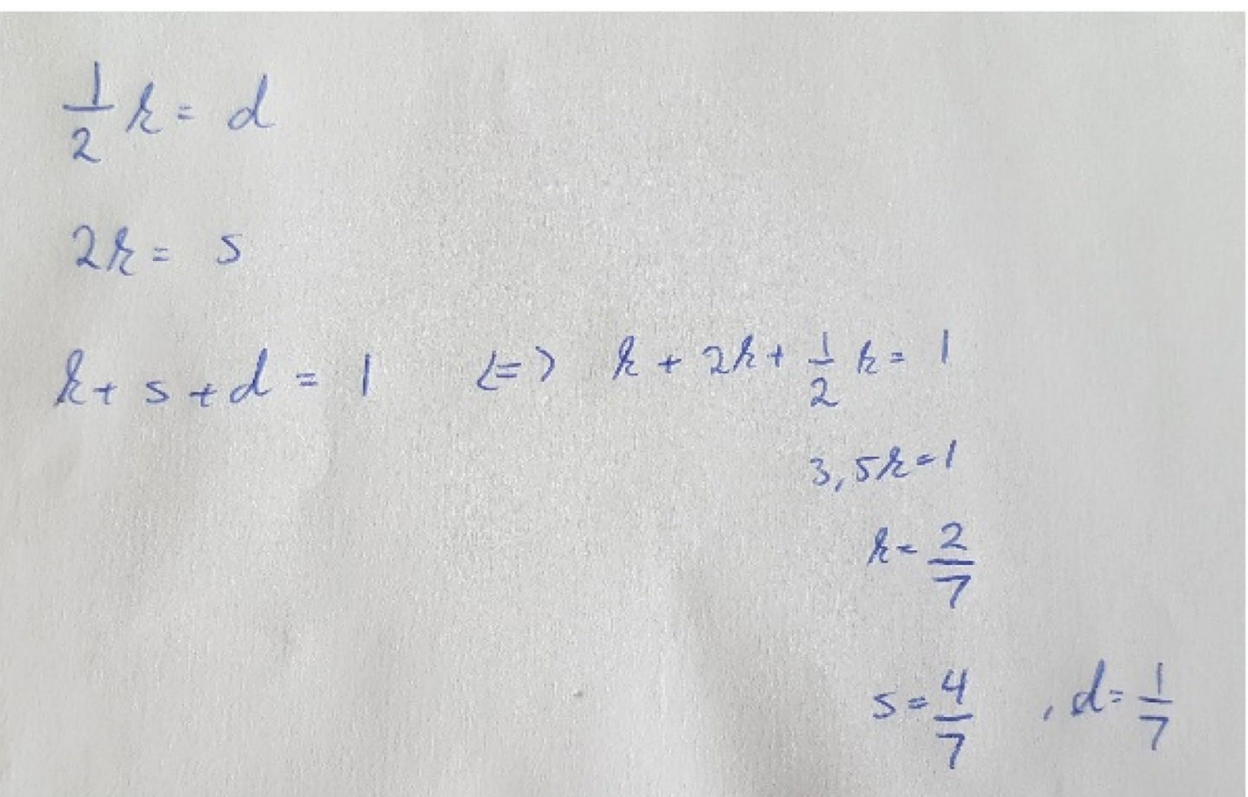

The students concluded that this was the right result. One of the students said: "The wife gets $\frac{2}{7}$, the daughter gets $\frac{1}{7}$, and the son gets $\frac{4}{7}$. This is the right result I guess". However, this solution takes into account only the ratio between the wife, son and daughter, and not the share of the inheritance each person was promised. The students in the expert group mentioned this inconsistency a few times, but as one of the students said: "this is a bit weird, but I guess this is how you solve the problem".

Novice students We identified three approaches for the novice groups.

As did the experts, all four novice groups first read the problem several times. However, unlike the experts, none of the novice groups discussed the information or constraints in the problem. Instead, they immediately started proposing possible solution strategies. The first approach all four novice groups attempted was some form of fraction expansion, followed by an empirically test to see if a more fine grained partition could make the inheritance division correct. The students would first set up a preliminary model, for instance imposing the constraints that the son would get more than the wife, and the wife would get more than the daughter. Then, they would adjust the model according to the results using bar charts, matrices or other heuristic approaches, and compare them to the conditions of the task. All four groups of students came up with at least three different partitions, before concluding that they were not able to build a model that satisfied all conditions of the task (see Fig. 3).

After concluding that the first approach did not satisfy all the conditions of the problem, all four novice groups immediately moved on to what we identified as a second approach. In the second approach, the novice students would use one of the son, the wife or daughter as a starting point based on

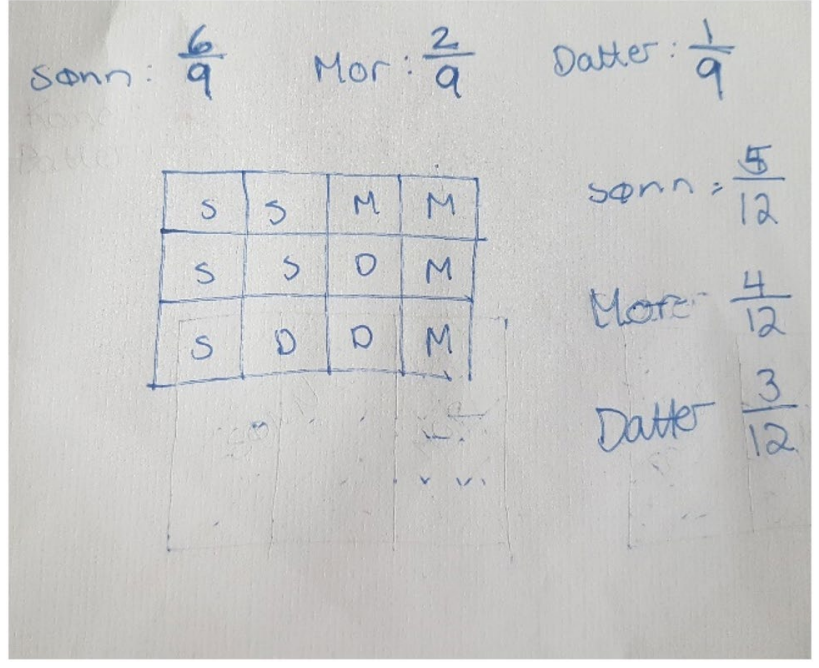

Fig. 3 Example of novices' model solution for the Roman problem

the information in the task, and then quantify what share of the inheritance the others would get. For instance, if the son would receive $\frac{2}{3}$ of the inheritance, then the wife would get $\frac{2}{9}$ and the daughter would get $\frac{1}{9}$. The students would then use the daughter or the wife as the starting point, respectively, and quantify how much the others would get. However, after trying different starting points, all four novice groups concluded that this approach would not provide a correct solution.

The third approach we observed for all four novice groups was similar to the expert group's second approach. The students wrote down and identified the ratios between the wife, the son and the daughter as the key constraints of the task. This approach was observed immediately after the novice 
students concluded their second approach was inappropriate, and it was also clear that this approach was inspired by the second approach. As one student said: "We have to take into account all constraints. At the same time. Not one by one. The son should get twice as much as the wife, and the wife should get twice as much as the daughter." However, unlike the expert students, the novice students did not explicitly formulate equations that represented the conditions of the problem. Instead, they reasoned more informally. As one student said: "the wife should get twice as much as the daughter, and the son should get twice as much as the wife. The daughter then gets one part, the wife two parts, and the son four parts. That gives us seven parts in total". All three novice groups concluded that this was the solution closest to the intentions of the will, but still not a satisfactory solution. After the third approach, three of the novice groups discussed the overall intentions of the will and which of their approaches was most in line with the wishes of the dying Roman. All three novice groups concluded that it was impossible to find a solution that was in full accordance with the will. However, all three groups also concluded that the main intention of the will was that the son should get more than the wife, and the wife should get more than the daughter.

\subsection{Problem 2: wrong arithmetic, but correct result}

Expert students The expert students approached the problem in two ways. First, the expert students read the problem, first individually and then out aloud. The experts then spent a few minutes talking about how "weird the expression was", while verifying that both sides of the equation were equal, and the proposed method was correct. The students quickly agreed on both the meaning and goal of the problem. As one student said: "oh, they've just placed the digits together, and we need to find out when fraction multiplication gives this kind of product." After verifying that the expression was indeed correct, the students proposed a hypothesis for which type of numbers this method was correct based on the example given. The students quickly mentioned that the sums of the digits in both the numerators and denominators were nine, and that nine was also a common factor of both 18 and 45. However, this hypothesis was not pursued further. Instead, the students quickly rejected the first approach and decided to represent the problem algebraically, which we have identified as their second approach.

After setting up the algebraic expression seen in Fig. 4, the students repeatedly stated that this expression wasn't appropriate. As one student said: "you can't use correct algebra on something that is incorrect. The left side is ok, but the right side is completely wrong". One of the students mentioned that they could have further identified constraints on each of the four "unknowns", but he quickly decided that such a pursuit was pointless as it was "not

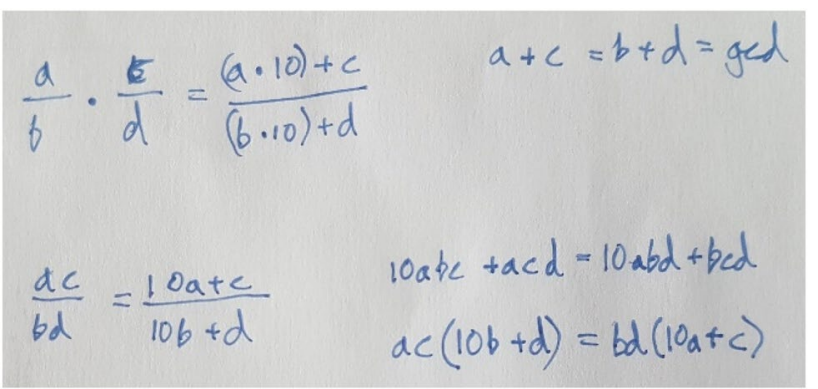

Fig. 4 Experts' algebraic solution for the Wrong arithmetic, but right result problem

correct mathematics". The students then concluded that they couldn't find any other solutions, as it couldn't be solved algebraically and it was difficult to generalize any sort of pattern from just one case.

Novice students Each of the four novice groups approached the problem in two ways. As with the Roman inheritance problem, all four novice groups first read the problem both individually and out loud. However, unlike the experts, the novice students did not explicitly discuss and agree on the meaning and goal of the problem. Instead, they seemed to spend a few minutes on their own trying to understand the problem. This period of apparent uncertainty was then interrupted by one of the students in the group proposing a particular solution strategy. For all four novice groups this involved a proposed hypothesis regarding the relationship between the numbers, which they refined empirically without considering the mathematical structure of the problem. For instance, the students explored commutativity and tried $\frac{8}{5} \times \frac{1}{4}=\frac{81}{54}$, they added the same numbers to denominators and numerators, and attempted to work with more or less randomly chosen fractions that, according to one student, were "in the same ballpark" as the fractions in the task. Common to all these hypotheses were that they were inferred from the specific numerical example in the problem text, and they were not based on any systematic investigation of the structural properties of the expression. One student, for example, evaluated the hypothesis according to "how close they came to giving an equal left and right side". The students switched back and forth between several different hypotheses, but did not explicitly consider how the right side of the expression was constructed mathematically. Eventually, all four novice groups concluded that this approach was not "fruitful", as one student said.

Eventually, all four novice groups rejected the first approach. Although there were some variations between the four groups, it seemed the second approach was an informal line of reasoning similar in structure to the novice students' third approach on the Roman inheritance problem. Furthermore, the second approach seemed to evolve 
out of the seemingly superficial hypotheses proposed in the first approach. As one student said, "We need to make things easier... we're just looking for connections between the numbers here, but there can so many of them." In the second approach, the novice students seemed to look for specific examples that would satisfy the conditions of the problem and thus identify possible structural relationships. For instance, three of the novice groups realized eventually that they could just "turn the fractions upside down and maintain the same ratio between them" as one student said. Two of the groups also listed several trivial solutions that satisfied the criterion $1 \times 1=1$. The main difference between the novices' first and second approaches, was that the first approach seemed to focus on identifying properties in the numbers given in the task, while the second approach seemed to focus on finding other examples that also satisfied the proposed method (see Fig. 5).

\subsection{Problem solving model}

During the orientation phase of both tasks, both the experts and novices first read the task instructions individually and aloud. Both groups of students seemed to prefer to read the problem first and gain an initial understanding of it before talking about it to the other students. However, after reading the problem carefully, either quietly or aloud, the rest of the orientation phase was different for the experts and

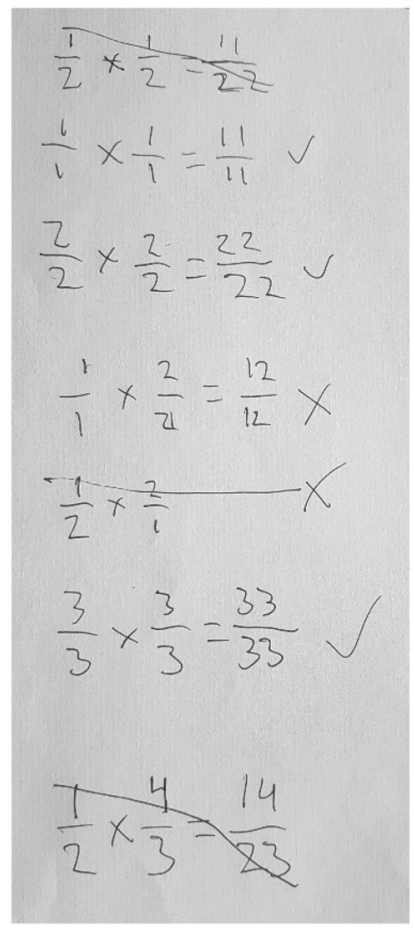

Fig. 5 Example of novices' empirical model solution to the Wrong arithmetic, but right result problem novices. While the experts wrote down and discussed the goals and conditions of the problems, seemingly to make sure everyone had the same understanding of the problem and its goal, the novices immediately began working on a solution strategy proposed by one of the students. Furthermore, after rejecting their first more informal approach, the experts went back to the orientation phase to make sure they all understood the problem correctly and had identified all the relevant conditions of the problem. There were also similarities and differences between the experts and novices in the organization and execution phases. For both problems, the experts first quickly proposed and rejected a hypothesis that seemed to be based on surface properties and incomplete constraints of the problems. For example, regarding problem two, there seemed to be no deeper analysis of the problem behind the first approach other than trying to identify common properties of the numbers on both sides of the equation sign. Similarly, the novices also first proposed hypotheses that seemed to be based on surface properties and incomplete constraints of the two problems. However, after rejecting the first approach, the experts then quickly sought a generalized and formalized solution, by representing and applying algebraic expressions and equations. The novices, on the other hand, continued to formulate hypotheses that they tested empirically, or they looked for numerical examples that satisfied given constraints of the problems. Finally, during the verification phase, there were also some noticeable differences between the two groups of students. The expert students quickly concluded, without any form of justification, that their first approach, for both problems, was incorrect. The students then similarly concluded quickly that their second approach was either correct or that the problem couldn't be solved, for problem 1 and problem 2 respectively. Unlike the expert students, who evaluated each approach quickly and conclusively after the organization and execution phase, the novices seemed to evaluate the approach continuously and gradually come to a conclusion regarding its correctness.

These observations are in line with much of the existing literature on expert vs. novice problem solvers (Lester \& Kehle, 2003; Schoenfeld, 1985a). The experts placed a greater focus on understanding the problem, global planning, and creating representations that captured the structural properties of the problems. The novices, on the other hand, tended to go directly from the problem text in search of solution strategies that could be productive. Furthermore, the novices tended to create representations of the problems that were either incomplete or focused on surface properties. We also noticed that the experts quickly determined whether or not a particular approach was correct, while the novices seemed to explore each approach to a much greater extent before assessing its validity. This could be a result of a more extensive knowledge base. How the two groups 
of students moved between the different problem solving phases is also similar to results in the literature regarding expert and novice problem solving. Schoenfeld (1985a) found, for example, that novices tend to spend much time on what he called the explore phase, which can be said to be an unstructured exploration of the problem analogous to orientation and organization. Experts, on the other hand, tend to display greater control and monitoring as they cycle more purposefully between the different problem solving phases. In this study, the experts' problem solving behavior seemed to consist of repeating cycles of orientation $\rightarrow$ organizing/ execution $\rightarrow$ verification. The novices, on the other hand, seemed to stick to cycling back and forth between the organizing/execution phase and the verification phase, after a single and initial orientation phase.

\subsection{Creativity model}

For the experts, we identified two approaches for each of the two problems. For the novices, we identified three approaches for the first problem and two approaches for the second problem. Immediately, a purely quantitative analysis would seem to indicate that the novices displayed greater cognitive flexibility during the problem solving process. However, a more detailed analysis reveals a more nuanced picture. For both problems, the experts' first approach seemed to be unstructured exploration based on either surface or an incomplete set of properties of the problem. The second approach, on the other hand, for both problems, was a more general and structured approach, where all the relational properties of the problem were represented using algebraic equations. For example, the experts' first approach to the Roman inheritance problem seemed to conclude that the wife's part of the inheritance would simply be the midpoint of the two different situations described in the will. The second approach, on the other hand, was an equation that seemingly covered all the relational properties described in the problem. The experts' work on both problems indicates a prominent mental shift between the first and the second approaches. It seems they were able to quickly break away from an inappropriate approach and instead pursue a more appropriate approach. Furthermore, the second approach is vastly different from the first approach in terms of both assumptions and strategies. As Nijstad et al. (2010) pointed out, sudden switching between remote mental sets-such as assumptions and strategies within a particular approach-is a key feature of cognitive flexibility. The novices, on the other hand, seemed to switch between approaches that were related to each other. For example, the novices' two approaches on the Wrong arithmetic, but correct result problem were both based on unstructured exploration around arithmetic properties. This pattern indicates that although the novices were able to break away from unproductive approaches, the closely related approaches indicate less cognitive flexibility than that shown by the experts. This interpretation is in line with much of the relevant literature which concludes that extensive knowledge is positively associated with flexible problem solving (Ionescu, 2012).

Turning to the issue of cognitive fixation, we observed several incidents of ostensible impasses from which the experts and novices were unable to break. For both problems, the novices stuck to empirical investigations of hypotheses and informal reasoning. Although the novices shifted fluidly between different assumptions and strategies for both problems, the fact that they stuck to a particular set of approaches, indicates to some extent the presence of algorithmic fixation (Haylock, 1987). Although algorithmic fixation primarily refers to the inappropriate continued use of a particular algorithm, this kind of fixation also includes a more general predisposition to solve a problem in a specific manner even though better or more appropriate methods of solving the problem exist. Creating, for example, algebraic representations for both problems, in particular the second problem, would have helped the novices determine the relevant structural properties. The experts also experienced incidents of prolonged impasse that could indicate cognitive fixations. However, unlike the novices who displayed tendencies of algorithmic fixation, the experts seemed to primarily display tendencies of content universe fixation (Haylock, 1987). Working on the first problem, the experts concluded quickly that their second approach was "the correct solution", as one student said, even though the constraints of the problem were not fully exhaustive and the ill-defined nature of the problem allowed multiple interpretations. For the second problem, the experts repeatedly stated that the algebraic expression (see Fig. 4) they had created was not appropriate, as they believe you could not apply "correct algebra on something that is incorrect", as one student said. However, within the context of the problem, creating an equation that captures all the relevant structural properties is perfectly appropriate. In fact, analyzing the algebraic expression would have help the students' identify the constraints of each digit. Overall though, the findings in the context of creativity is also in line with much of the literature. Both the experts and the novices displayed both flexibility and fixation during the problem solving process-although somewhat differently.

\subsection{How students gained insight}

Immediately, it would appear that the findings in this study are in line with much of the literature on expert and novice problem solving. Furthermore, both the experts' and the novices' work seemed to progress largely in a stepwise manner, as described and explained both by the problem solving model utilized in this study (Lester, 1985) and the 
analytic view of insight (Weisberg, 2015). One instance of this aspect can be seen in the novices' work on the first problem. While their second approach was premised only on a single constraint of the problem, their third approach took into account all the relational properties between the wife, the daughter and the son simultaneously. In this instance, the novice students' clearly modified their approach in a gradual and stepwise manner and further insight was gained as a result. A second important instance can be found in the experts' work. For both problems, the experts returned to the orientation phase after their first approach, and then produced a new and more effective approach. This chain of events indicates that the experts' first ineffective approach and return to the orientation phase somehow led to a productive mental restructuring of the problem-or greater insight in other words- which in turn resulted in a more effective approach.

However, a more finely-grained scrutiny of the students' work reveals several limitations of the problem solving model. One such discrepancy is the emphasis on past experiences during problem solving (Liljedahl et al., 2016). Problem solving models (Lester, 1985; Pólya, 1949; Schoenfeld, 1985a), and the analytic view of insight (Weisberg, 2015), highlight the importance of past experiences during problem solving and argue that insight is a consequence of matching the problem with information in memory. In this study, we did not observe a single incident in which either group explicitly referenced past experiences or compared the problem to other problems. It could be argued that the ill-defined structure of the problems themselves was unfamiliar, but it is still noticeable that neither group of students performed any sort overt assessment of familiarity with the task (Lester, 1985).

Another ostensible discrepancy can be found in the novices' many approaches to the problems. Although the novices did not move between the different problem solving phases to the same extent as the experts, they did not stick to one particular approach "come hell or high water"-as Schoenfeld (1985a) observed to be common among novice problem solvers. Instead, the novices moved seemingly effortlessly between different approaches, constantly adapting to the ambiguity of the ill-defined problems. This behavior is a clear indication of cognitive flexibility (Ionescu, 2012). Furthermore, each of these apparent mental restructurings of the problems seemed to follow small impasses in the problem solving process-as predicted by the Gestaltists (Weisberg, 2015).

Insight as a consequence of impasses and sudden mental restructuring, as opposed to a stepwise and conscious process, was even more prominent in the experts' work. The experts' work on both problems indicates a significant mental shift between the first and the second approach. After trying and concluding that their first and more informal approach was inappropriate, the experts quickly decided to pursue a completely different and more structured approach. Although this behavior can be projected on to the four phases of the problem solving model (Lester, 1985), as seen earlier, the model itself cannot qualitatively explain the drastic shift in terms of assumptions and strategies. The experts' second approach was in no way a further refinement of their first approach, and they did not explicitly reference past experiences. Instead, it seemed the second approach appeared suddenly, unconsciously and as a response to the failure of the first approach. This chain of events is similar to what Ohlsson (2011) refers to as the insight sequence, which describes insight as something gained after an attempted solution fails and a sudden and meaningful mental restructuring is required. After an impasse has occurred, insight is gained after dealing with the problem from a completely novel perspective.

Finally, our analyses also indicate occurrences in which both groups of students failed to gain insight. For example, while the novices applied mostly empirical and informal reasoning, the experts sought generalized and formalized solutions. Although much of the literature explains this as a consequence of the experts' more extensive knowledge base (Lester \& Kehle, 2003; Schoenfeld, 1985a), neither problem used in this study required advanced mathematics. The algebraic representations that the experts made use of were fairly simple and seemingly within the grasp of individuals who have taken at least upper secondary algebra. An alternative explanation can therefore be cognitive fixation (Haylock, 1987), in which individuals fail to abandon ineffective approaches and move beyond impasses. This was perhaps seen most clearly in the experts' work on the second problem. After creating an algebraic representation of the structural properties of the problem, the experts quickly rejected, in unison, the approach as inappropriate. We propose that this is a clear example of an unnecessary restriction to an insufficient range of elements (Haylock, 1987). In other words, the experts imposed an unnecessary set of restrictions on to the problem solving process based on their conceptions of the situation, rather than the properties of the problem itself. Now, it can be argued that this fixation can be linked to the experts' past experiences. However, the problem solving model, and the analytic view of insight, do not explain or describe how the problem solver can break away from established mental sets. In fact, the problem solving model, and the analytic view of insight, emphasize the use of prior knowledge and reliance on past experiences when first attacking a problem (Liljedahl, 2016). When facing a new problem, in particular an ill-defined problem such as those made use of in this study, the focus on past experiences could actually be a hindrance to making progress (Weisberg, 2015). 


\section{Final thoughts}

In this study, we aimed to integrate two different views on insight during problem solving, and explore how they each highlight different aspects of the problem solving process. Looking back, applying both problem solving and creativity models on to the experts' and novices' work reveals and explains different aspects of the students' problem solving processes. While the problem solving model helps us analyze and understand parts of the problem solving process, there are crucial aspects of the students' work that it does not explain. In this study, we observed what we claim to be the occurrence of cognitive flexibility, cognitive fixation, and more importantly, sudden, and seemingly unconscious, insight during the problem solving process - for both experts and novices. The results of this study therefore dovetail with what the Gestaltists said all along: Sudden and unconscious insight seems to be crucial to the problem solving process, and the occurrence of such insight cannot be fully explained by standardized problem solving models and an analytic view of insight. Current researchers inspired by the Gestaltists have dubbed this understanding of insight as the special process view of insight (Ohlsson, 2011; Weisberg, 2015), as it asserts that the thought processes underlying insight are distinctly different from those thought processes underlying analytic thinking.

We suggest, based on the results of this study and the review of the relevant literature, that research into problem solving within mathematics education would benefit from adopting aspects of Gestalt inspired views of insight. Although we do not go as far as some who claim that adherence to any sort of heuristics can be a hindrance to the problem solving process, we do agree that there are no prescriptive heuristics for some of the more unconscious, yet highly important, cognitive aspects of problem solving (Liljedahl et al., 2016). So, what happens during the moment of insight or subconscious work? What is the source of creative thought? Although we do not fully understand mental restructuring and creative thought, Ohlsson (2011) has proposed redistribution theory as a Gestalt-inspired response. Here, the problem solver first creates an initial inappropriate representation of the problem. This particular interpretation activates one or more incorrect solutions, which the problem solver then works through. At some point, after working through the incorrect solutions, the problem solver reaches an impasse. It is at this point that the initial, and inappropriate, representation of the problem could be inhibited. This inhibition of the original representation of the problem might then result in a new representation of the problem, which causes the problem solver to realize that the problem can be thought of in a different way-in other words, a mental restructuring has occurred. Somewhat ironically, the Gestalt inspired method of problem solving can therefore also be said to rely heavily on past experience. What is entailed is not to match the problem with past experiences to find an appropriate solution, but rather to relax unnecessary constraints and inhibit knowledge that is not necessary. We propose that this line of reasoning can add to extant problem solving models in at least two ways, as follows: 1) Most problem solving models highlight the importance of assessing the familiarity of the problem (Lester, 1985; Liljedahl et al., 2016; Pólya, 1949; Schoenfeld, 1985a). However, the heuristic emphasis seems to be on identifying similarities between the problem at hand and past experiences. We suggest that identifying divergences between the problem at hand and past experiences is also important, as it may help the problem solver recognize unnecessary constraints. 2) Working through numerous incorrect approaches and solutions can be helpful to the overall problem solving process, as it may lead to an impasse and a subsequent more appropriate restructuring of the problem. We suggest that problem solving models should also emphasize the value of working hard on problems for an extended period of time, and even failed attempts.

Funding Open access funding provided by UiT The Arctic University of Norway (incl University Hospital of North Norway).

Open Access This article is licensed under a Creative Commons Attribution 4.0 International License, which permits use, sharing, adaptation, distribution and reproduction in any medium or format, as long as you give appropriate credit to the original author(s) and the source, provide a link to the Creative Commons licence, and indicate if changes were made. The images or other third party material in this article are included in the article's Creative Commons licence, unless indicated otherwise in a credit line to the material. If material is not included in the article's Creative Commons licence and your intended use is not permitted by statutory regulation or exceeds the permitted use, you will need to obtain permission directly from the copyright holder. To view a copy of this licence, visit http://creativecommons.org/licenses/by/4.0/.

\section{References}

Beghetto, R. A., \& Karwowski, M. (2019). Unfreezing creativity: A dynamic micro-longitudinal approach. In R. A. Beghetto \& G. E. Corazza (Eds.), Dynamic perspectives on creativity (pp. 7-25). Springer.

Bilalic, M., McLeod, P., \& Gobet, F. (2008). Inflexibility of expertsReality or myth? Quantifying the Einstellung effect in chess masters. Cognitive Psychology, 56, 73-102.

Corazza, G. E. (2016). Potential originality and effectiveness: The dynamic definition of creativity. Creativity Research Journal, $28,258-267$. 
Dreyfus, H. L., \& Dreyfus, S. E. (2005). Peripheral vision: Expertise in real world contexts. Organization Studies, 26(5), 779-792.

Elgrably, H. \& Leikin, R. (2021). Creativity as a function of problemsolving expertise: posing new problems through investigations. ZDM Mathematics Education, 53, 891-904.

Ericsson, K. A., \& Lehmann, A. C. (1996). Expert and exceptional performance: Evidence of maximal adaptation to task constraints. Annual Review of Psychology, 47(1), 273-305.

Flavell, J. H., \& Wellman, H. (1977). Metamemory. In R. Kail \& J. Hagen (Eds.), Perspectives on the development of memory and cognition. Lawrence Erlbaum Associates.

Glaser, R. (1987). Thoughts on expertise. In C. Schooler \& W. Schaie (Eds.), Cognitive functioning and social structure over the lifecourse (pp. 81-94). Ablex.

Haavold, P., Sriraman, B., \& Lee, K. H. (2020). Creativity in mathematics education. In S. Lerman (Ed.), Encyclopedia of mathematics education (2nd ed., pp. 145-154). Springer.

Hadamard, J. W. (1945). Essay on the psychology of invention in the mathematical field. Princeton University Press.

Haylock, D. W. (1987). A framework for assessing mathematical creativity in school children. Educational Studies in Mathematics, 18(1), 59-74.

Hoffman, R. R. (1998). How can expertise be defined? Implications of research from cognitive psychology. In R. Williams, W. Faulker, \& J. Fleck (Eds.), Exploring expertise (pp. 81-100). Macmillan.

Ionescu, T. (2012). Exploring the nature of cognitive flexibility. New Ideas in Psychology, 30(2), 190-200.

Kitchener, K. S. (1983). Cognition, metacognition, and epistemic cognition: A three-level model of cognitive processing. Human Development, 4, 222-232.

Krutetskii, V. A. (1976). The psychology of mathematical abilities in schoolchildren. University of Chicago Press.

Lester, F. K. (1985). Methodological considerations in research on mathematical problem solving. In E. A. Silver (Ed.), Teaching and learning mathematical problem solving. Multiple research perspectives (pp. 41-70). Hillsdale: Lawrence Erlbaum Associates.

Lester, F. K. (2013). Thoughts about research on mathematical problem-solving instruction. The Mathematics Enthusiast, 10(1), 245-278.

Lester, F. K., \& Kehle, P. E. (2003). From problem solving to modeling: The evolution of thinking about research on complex mathematical activity. In R. Lesh \& H. Doerr (Eds.), Beyond constructivism: Models and modeling perspectives on mathematics problem solving, learning and teaching (pp. 501-518). Lawrence Erlbaum Associates.

Liljedahl, P., Santos-Trigo, M., Malaspina, U., \& Bruder, R. (2016). Problem solving in mathematics education. Springer International Publishing.
Mayring, P. (2015). Qualitative content analysis: Theoretical background and procedures. In A. Bikner-Ahsbahs, C. Knipping, \& $\mathrm{N}$. Presmeg (Eds.), Approaches to qualitative research in mathematics education. Examples of methodology and methods (pp. 365-380). Springer.

National Research Council. (2000). How people learn: Brain, mind, experience, and school. National Academy Press.

Nijstad, B. A., De Dreu, C. K., Rietzschel, E. F., \& Baas, M. (2010). The dual pathway to creativity model: Creative ideation as a function of flexibility and persistence. European Review of Social Psychology, 21(1), 34-77.

Ohlsson, S. (2011). Deep learning: How the mind overrides experience. Cambridge University Press.

Poincaré, H. (1948). Science and method. Dover.

Pólya, G. (1949). How to solve it. Princeton University Press.

Schoenfeld, A. H. (1985a). Mathematical problem solving. Academic Press.

Schoenfeld, A. H. (1985b). Making sense of "out loud" problemsolving protocols. The Journal of Mathematical Behavior, 4(2), 171-191.

Simon, M. A. (2019). Analyzing qualitative data in mathematics education. In K. R. Leatham (Ed.), Designing, conducting, and publishing quality research in mathematics education (pp. 111-123). Springer.

Simon, D. P., \& Simon, H. A. (1978). Individual differences in solving physics problems. In R. Siegler (Ed.), Children's thinking: What develops? (pp. 325-348). Lawrence Erlbaum Associates.

Sriraman, B. \& Dickman, B. (2017). Mathematical pathologies as pathways into creativity. ZDM Mathematics Education, 49(1), 137-145.

Sternberg, R. J., \& Davidson, J. E. (1995). The nature of insight. MIT Press.

Wallas, G. (1926). The art of thought. New York, NY: Harcort Brace and World.

Webb, M. E., Little, D. R., \& Cropper, S. J. (2016). Insight is not in the problem: Investigating insight in problem solving across task types. Frontiers in Psychology, 7, 1-13.

Weisberg, R. W. (2015). Toward an integrated theory of insight in problem solving. Thinking \& Reasoning, 21(1), 5-39.

Wertheimer, M. (1959). Productive thinking (Enlarged Edition). Harper and Brothers.

Publisher's Note Springer Nature remains neutral with regard to jurisdictional claims in published maps and institutional affiliations. 